\title{
Effects of prior discriminative stimulus and reinforcer presentation on acquisition of instrumental responding in rats*
}

\author{
JOHN H. HULL and JAMES S. MYER† \\ Kent State University, Kent, Ohio
}

\begin{abstract}
Independent groups of rats underwent various pretraining procedures involving key ligint illumination and presentation of food before introduction of instrumental conditioning in whicii food reinforcement was immediately contingent upon pressing the illuminated key. Key illumination followed immediately by food presentation produced facilitation of subsequent acquisition of instrumental responding which increased with $0,10,50$, and 200 pretraining trials; the facilitative effect of 1,000 pairings of key illumination and food was no greater than that of 200 pairings. The facilitative effect of key illumination and food presentation upon subsequent instrumental learning was the same regardless of whether food presentations in pretraining immediately accompanined illumination offset or were delayed for 8 sec. Food presentations alone on 10,50, or 200 pretraining trials facilitated subsequent instrumental learning less than equivalent numbers of presentations of illumination and food, but 200 presentations of fuod alone facilitated subsequent instrumental learning more than 0 pretraining trials.
\end{abstract}

There have been several studies of the effects of response-independent pairings of a stimulus and reinforcer upon subsequent appetitive instrumental discrimination learning. In a typical experiment, Bower and Grusec (1964) trained rats to press a lever for water. The lever then was removed and two tones were presented alternately. Water was periodically delivered during one tone presentation and not the other. The lever was then reintroduced, and discriminative instrumental training with the successively presented stimuli began. For some animals, response-contingent water reinforcement was delivered during presentation of the tone previously associated with water, and for others the discrimination was reversed. The former group developed appropriate discriminative responding more rapidly than did the group for which the stimulus relations were reversed. Mellgren and Ost (1969) confirmed this finding under similar conditions, and, by employing a control group which received water presentations during both stimuli before discriminative instrumental training, showed that the effect was due both to facilitation of performance in the group which experienced consistent stimulus-reinforcer relations and interference in the group in which the relations were reversed.

A thorough series of studies by Trapold and his collaborators (Trapold \& Winokur, 1967; see Trapold \& Overmier, 1972) has generally confirmed these findings. However, Trapold, Lawton, Dick, and Gross (1968)

*This research was supported by Grant GB-31939 from the National Science Foundation. Portions of the research were submitted by the first author as a thesis in partial fulfillment of the requirements of the Master of Arts degree at Kent State University.

†R equests for reprints should be sent to James S. Myer, Department of Psychology, Kent State University, Kent, Ohio 44240 . failed to find a facilitation of discriminative instrumental responding by previous association of the positive stimulus with food; and Hyde, Trapold, and Gross (1968) found a facilitative effect of a signal for reinforcement upon subsequent acquisition of discriminative instrumental responding but variation of magnitude of prior noncontingent reinforcement presentations had no effect on performance in the discriminative instrumental situation.

In the described experiments, response-independent presentations of discriminative and reinforcing stimuli were preceded by acquisition of the instrumental response, and the effects of the stimulus presentations on subsequent formation of a discrimination were studied. The effect of prior stimulus exposure on acquisition of a positively reinforced instrumental response has seldom been studied, probably because shaping procedures involving reinforcement of successive approximations of the instrumental response typically are used to establish responding. The experiment reported here was conducted to analyze the effects of prior exposure to pairings of the discriminative stimulus and reinforcer and of reinforcement alone on subsequent acquisition of a food-reinforced response in rats.

Previous research (Myer \& Hull, 1974) has shown that rats will learn to press a periodically illuminated key when food presentation is contingent on that response, even if no hand-shaping or autoshaping procedure is employed. In the present study, independent groups of rats were exposed to different numbers of pairings of key illumination and food presentation, with either a 0 -sec or 8-sec interval between key illumination termination and food presentation. They then underwent a discrete trials instrumental conditioning procedure in which food was delivered only if the 
illuminated key was pressed within a fixed time period. It was expected that greater numbers of prior light-food pairings would result in faster acquisition of the instrumental response, but that an 8-sec delay between light offset and food presentation during the first pinase would retard acquisition of the instrumental response, relative to no delay between light offset and food presentation during preinstrumental training. Additional rats were exposed to different numbers of presentations of food alone prior to instrumental training. If the light-food contingency prior to instrumental training is a potent determinant of rate of acquisition of the instrumental response, presentation of food alone should not facilitate acquisition of instrumental responding as much as pairings of light and food prior to instrumental training.

\section{METHOD}

\section{Subjects}

The Ss were 88 adult female Long-Evans rats obtained from Huntingdon Farms. The rats were maintained at $80 \%$ ad lib body weight under conditions of constant artificial illumination, and were housed in the experimental room in individual cages with continuous access to water. White masking noise was constantly present in the experimental room during experimentation.

\begin{abstract}
Apparatus
Four $20.0 \times 23.5 \times 19.0 \mathrm{~cm}$ high Scientific Prototype rodent test cages were enclosed in sound-attenuating chambers containing a $25-\mathrm{W}$ red incandescent house light. A flat, transparent Plexiglas key was mounted flush with the outside of each of the narrower walls of each test cage over a $2.54-\mathrm{cm}$ diam hole centered on the wall $3.0 \mathrm{~cm}$ above the floor. A small light behind each key was controlled by programming equipment in an adjacent room. Reinforcers were $20-\mathrm{mg}$ Noyes laboratory food pellets automatically dispensed into a cup located on the floor of one of the long walls of the cage. Two response keys were employed, and the response keys and food cup were placed far apart to minimize the possibility of adventitious reinforcement of keypresses prior to the instrumental phase of the experiment.
\end{abstract}

\section{Procedure}

Each rat was given a 55-min adaptation session in its test cage on the day before the first experimental session. On the adaptation day, there were 10 food pellets in the food cup of the test cage. The adaptation session was conducted to assure that the effects of the manipulations of light and food presentations on subsequent instrumental responding could not be attributed solely to familiarity with the experimental apparatus.

On the day following adaptation, four groups of eight rats received $10,50,200$, or 1000 light-food pairings, respectively. Pairings of light and food were administered on a variable time schedule, with an average of $30 \mathrm{sec}$ between trials. Only one key was illuminated on each trial. Each of the two keys was illuminated on one-half of the trials, with trials distributed across the keys in an irregular sequence. On each trial a key was illuminated for $10 \mathrm{sec}$ and a food pellet was presented when key illumination terminated, regardless of the rats' behavior. Presses that occurred during the $10-\mathrm{sec}$ light presentation had no experimentally programmed consequences. Rats in the 1000 pairings group received 200 light-food pairings per day for 5 days.

Three additional groups of eight rats received 10,50 , or 200 light-food pairings, respectively, with a procedure identical to that employed with the first four groups except that there was an 8-sec delay of food presentation after termination of each 10 -sec period of key illumination. Ten, 50 , or 200 food pellets were presented to another three groups of eight rats on the same 30 -sec variable time schedule employed with the other groups, but no key light was illuminated at any time.

For eight rats in an eleventh group, an instrumental conditioning procedure was introduced on the day after the adaptation session, with no intervening exposure to presentation of light or food. The same instrumental conditioning procedure was initiated for the other 10 groups on the day after their last sessions with response-independent pairings of light and food, or presentation of food alone.

The instrumental conditioning procedure was identical to the procedure previously employed except for the reinforcement contingencies and the duration of key light presentation. In instrumental conditioning, a food pellet was delivered and key illumination terminated immediately when the rat pressed the key. If no keypress occurred within $10 \mathrm{sec}$, key illumination terminated but no food pellet was delivered. Rats in all groups received 200 instrumental conditioning trials each day for 4 successive days.

\section{RESULTS AND DISCUSSION}

In Fig. 1, the first panel shows the mean number of instrumental responses on the first day of instrumental conditioning for all experimental groups. Panels 2, 3, and 4 present corresponding data for Instrumental Conditioning Days 2, 3, and 4, respectively. Repeated measures analyses of variance, eaci including data from the group which had no key light or food presentations before instrumental conditioning, indicated acquisition of the instrumental response was more rapid as the number of prior food presentations was increased, regardless of whether such food was presented immediately after key light offset $(F=5.99, d f=4 / 35$, $\mathrm{p}<.01) .8 \mathrm{sec}$ after key light of fset $(\mathrm{F}=3.55, \mathrm{df}=$ $3 / 28, p<.05$ ), or if nonsignaled food presentations were given $(F=4.12$, $\mathrm{df}=3 / 28, \mathrm{p}<.05)$. However, a Newman-Keuls test indicated that increasing the number of 0 -sec delay light-food pairings from 200 to $1000 \mathrm{had}$ no additional facilitative effect.

A 3 by 3 repeated measures analysis of variance was used to compare the instrumental performance of all groups receiving 10,50 , or 200 food presentations prior to instrumental conditioning. The analysis is summarized in Table 1. A Newman-Keuls test indicated that the significant effect of type of pretraining resulted from poorer acquisition of the instrumental response by those groups which received presentations of food alone prior to instrumental training as compared with corresponding groups which received light-food pairings.

In the present experiment, increasing the number of pairings of illumination of the key light with food facilitated subsequent acquisition of food-reinforced responding to an illuminated key, even if food presentation was delayed for $8 \mathrm{sec}$ after termination of illumination of the key. This finding indicates that close temporal contiguity of key light illumination and 


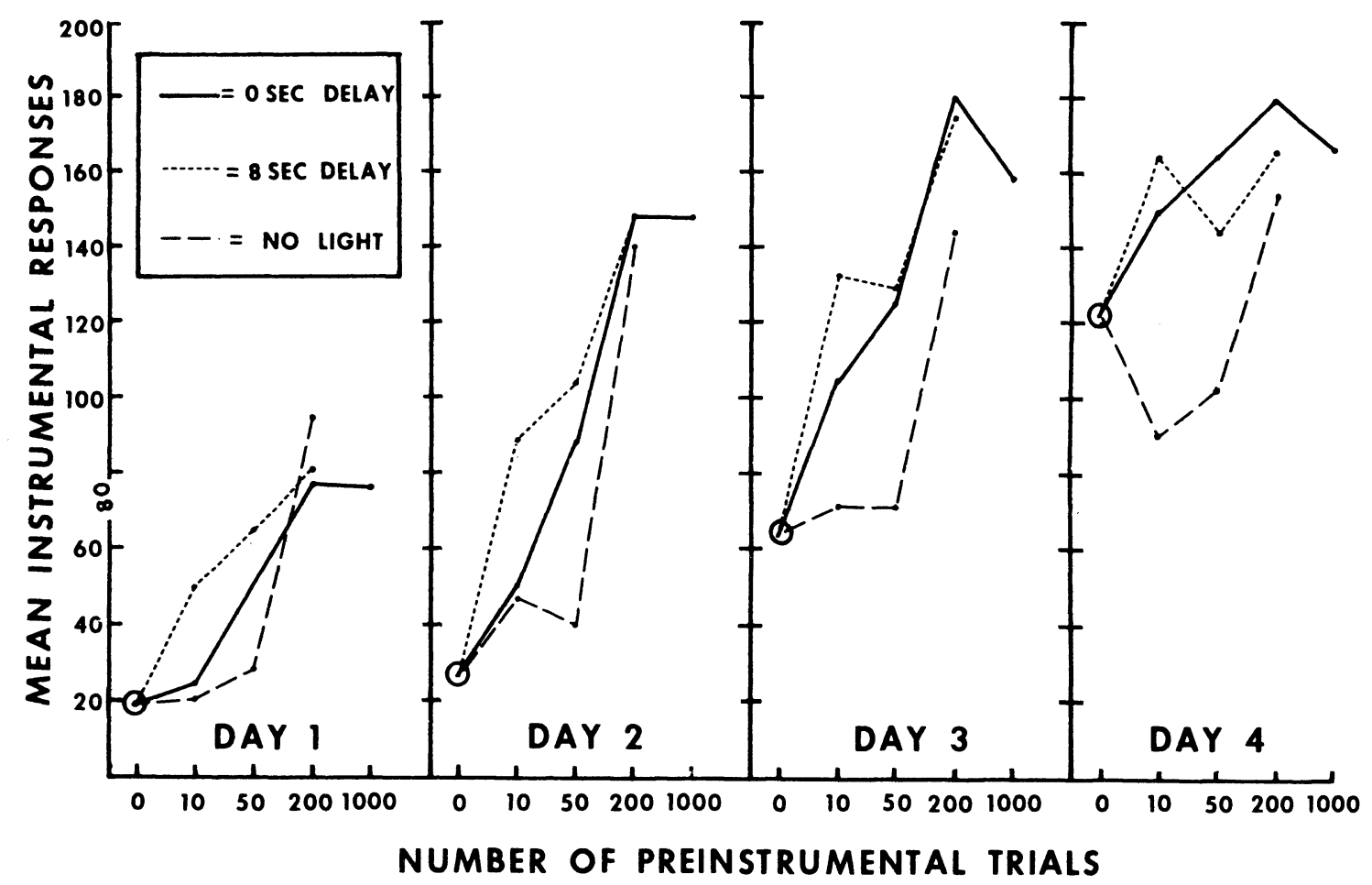

Fig. 1. Mean instrumental responses as a function of number of preinstrumental trials for groups which had food presentations 0 or $8 \mathrm{sec}$ af ter key light offset, or food presentations alone, during preinstrumental trials.

delivery of food is not necessary to produce the facilitative effects of presentation of light and food upon subsequent instrumental responding. This failure to demonstrate an effect of delay of reinforcement is reminiscent of a similar failure of Hyde et al (1968) to find an effect of magnitude of reinforcement. Together, these results suggest that the effect of stimulus pairings upon subsequent acquisition of instrumental responding is not as sensitive to variations of parameters of reinforcement as is conditioning in more traditionally studied situations.

Presentations of food alone during pretraining also facilitated subsequent acquisition of instrumental responding, although such presentations were not as effective as presentations of both light and food. All

Table 1

Summary Table: 3 by 3 Repeated Measures ANOVA

\begin{tabular}{lrrc}
\hline Source & \multicolumn{1}{c}{ SS } & df & F \\
\hline Number of Trials (N) & 173,998 & 2 & $9.22 * *$ \\
Type of Pretraining (P) & 69,976 & 2 & $3.71 *$ \\
N by P & 26,342 & 4 & $<1$ \\
Error & 594,339 & 63 & \\
Days (D) & 337,949 & 3 & $125.02 * *$ \\
N by D & 20,917 & 6 & $3.87 * *$ \\
P by D & 15,206 & 6 & $2.81 * *$ \\
N by P by D & 6,203 & 12 & $<1$ \\
Error & 170,297 & 189 & \\
Total & $1,415,227$ & 287 & \\
${ }^{*} p<.05$ & $* * p<.001$ &
\end{tabular}

${ }^{*} p<.05 \quad{ }^{* *} p<.001$ groups had 55-min exposures to the experimental apparatus prior to the beginning of any experimental procedures. It is, therefore, unlikely that the facilitation of acquisition of instrumental responding by the group given 200 presentations of food alone before instrumental training was solely due to familiarization with the experimental apparatus.

Inspection of the preinstrumental training data for groups which had light-food pairings with a 0 - or 8 -sec delay between light offset and food presentation indicated that some responding during key light illuminations did occur. However, such responding very rapidly decreased across trials for all groups, regardless of the light-food relationship. It seems paradoxical that the 200- and 1000-trial groups which were responding very little (approximately $4 \%$ of the key light illuminations) at the end of their preinstrumental training nevertheless acquired the instrumental response faster than did the 10-trial groups which were responding comparatively often (approximately 20\% of the key illuminations) during preinstrumental training. These observations indicate that more rapid acquisition of instrumental responding cannot be attributed to a higher rate of keypressing at the beginning of instrumental training in those groups which received larger numbers of preinstrumental light-food pairings.

The present findings contrast with the report of Engberg, Hansen, Welker, and Thomas (1972) that prior food presentations retarded subsequent acquisition of a food-reinforced keypecking response by pigeons. In the 
experiment reported here, food presentations with or without presentation of the stimulus light facilitated subsequent acquisition of the instrumental response. There were numerous procedural differences between the Engberg et al study and the experiment reported here. Furthermore, there is mounting evidence that the behavior studied by Engberg et al, keypecking for food by pigeons, may be rather uniquely influenced by variations in stimulus-reinforcer relationships prior to instrumental training (Gamzu \& Schwam, 1973; Myer \& Hull, 1974). It is evident that a considerably greater range of stimulus conditions, responses, and species must be studied before broad generalizations can be drawn about the effects of prior experience with discriminative stimuli and reinforcers upon subsequent acquisition of appetitively motivated instrumental responding.

\section{REFERENCES}

Bower, G., \& Grusec, T. Effect of prior Pavlovian discrimination training upon learning an operant discrimination. Journal of the Experimental Analysis of Behavior, 1964, 7, 401-404.

Engberg, L. A., Hansen, G., Welker, R. L., \& Thomas, D. R.
Acquisition of key-pecking via autoshaping as a function of prior experience: "Learned laziness"? Science, 1973, 178, $10002-1004$

Gamzu, E., \& Schwam, E. The generality of autoshaping: The squirrel monkey doesn't peck. Paper presented at the meeting of the Eastern Psychological Association, Washington, D. C., 1973.

Hyde, T. S., Trapold, M. A., \& Gross, D. M. Facilitative effect of a CS for reinforcement upon instrumental responding as a function of reinforcement magnitude: A test of incentive-motivation theory. Journal of Experimental Psychology, 1968, 76, 423-428.

Mellgren, R. L., \& Ost, J. W. P. Transfer of Pavlovian differential conditioning to an operant discrimination. Journal of Comparative \& Physiological Psychology, 1969, 67, 390-394.

Myer, J. S., \& Hull, J. H. Auto shaping and instrumental learning in the rat. Journat of Comparative \& Physiological in the rat. Journat of $\mathrm{C}$.

Trapold, M. A., Lawton, G. W., Dick, R. A., \& Gross, D. M. Transfer of training from differential classical to differential instrumental conditioning. Journal of Experimental Psychology, 1968, 76, 568-573.

Trapold, M. A., \& Overmier, J. B. The second learning process in instrumental learning. In A. H. Black \& W. F. Prokasy (Eds.), Classical conditioning II. Current theory and research. New York: Appleton-Century-Crofts, 1972.

Trapold, M. A., \& Winokur, S. Transfer from classical conditioning and extinction to acquisition, extinction, and stimulus generalization of a positively reinforced instrumental response. Journal of Experimental Psychology, 1967, 73, 517-525.

(Received for publication July 25, 1974.) 